\title{
Estimation of Land Surface Temperature Using Spatial Interpolation and Satellite-Derived Surface Emissivity
}

\author{
J. S. Yang*, Y. Q. Wang and P. V. August \\ Department of Natural Resources Science, University of Rhode Island, One Greenhouse Road, Kingston, RI 02881, USA
}

\begin{abstract}
This study presents a method to estimate land surface temperature (LST) by calibrating spatial interpolation using satellite-derived surface emissivity. Four spatial interpolation methods including Inverse Distance Weighting (IDW), Spline, Kriging, and Cokriging were tested to interpolate LST in southern New England using ground temperatures measured at national weather stations (NWS) in summer 2001. The performance of each interpolation method was evaluated using field measurements collected in a mixed forest in Connecticut during the study period. Kriging is recommended for LST interpolation when surface emissivity data are not available. By analyzing the field data, we found that the maximum daily interpolation error occurred in the early morning and the minimum error occurred at around hour 18:00. Validation result shows that the accuracy of spatial interpolation of LST was much improved after being calibrated by satellite-derived surface emissivity. The average interpolation error reduced from $10{ }^{\circ} \mathrm{C}$ before calibration to $1.56{ }^{\circ} \mathrm{C}$ after calibration.
\end{abstract}

Keywords: Land surface temperature, spatial interpolation, surface emissivity

\section{Introduction}

Land surface temperature (LST) is usually measured at a system of first, second, and third order national weather stations (NWS) in the United States. Most isometric LST maps are interpolated from measurements at these sample locations using different spatial interpolation methods. The interpolation of climatic data from sparsely stationed network has been a focus of research for a long time (Hughes, 1982; Phillips et al., 1992; Price et al., 2000; Vizuete et al., 2002). There are a number of deterministic and geostatistical interpolation methods to estimate the values in between sampling locations. For example, Inverse Distance Weighting (IDW) (Willmott et al., 1985) and Spline (Wahba, 1981) have been used to interpolate air temperature and its anomalies. The Kriging technique has been applied to estimate monthly ozone exposure and summer frost in growing season (Lefohn et al., 1988; Lindkvist and Lindqvist, 1997), and Cokriging for estimation of surface air temperature (Ishida and Kawashima, 1992).

Depending on the spatial attributes of the data, interpolation accuracies vary widely among different spatial interpolation methods. For example, significant differences were found among the temperatures estimated using different interpolation methods in Eastern Texas (Vizuete et al., 2002). Therefore, the selection of spatial interpolation method is especially important in the region where data collection is sparse and variable changes are significant over short spatial distances. Furthermore, unlike other climatic variables such as precipitation and solar radiation, the accuracy of LST estimation is

\footnotetext{
* Corresponding author: jyan1185@mail.uril.edu
}

strongly affected by the surface capability of emitting radiance (Synder et al., 1998). Many algorithms for estimating LST from satellite data are based upon the assumption that ground surface acts as a blackbody (emissivity equals one). In fact, most Earth's surfaces emit only a fraction of the energy emitted by a blackbody at the same temperature. The emission capacity of a land surface, compared to that of a blackbody, is referred to surface emissivity (Qin and Karnieli, 1999).

Surface emissivity for a large area is usually not available during the satellite overpass because it is very difficult to measure directly. Satellite-sensed thermal infrared (TIR) data is the major source for estimating surface emissivity for a region. For example, Moderate Resolution Imaging Spectroradiometer (MODIS) provides daily surface emissivity at $1 \times$ $1 \mathrm{~km}$ spatial resolution based on conventional image classification and laboratory measurements (Snyder et al., 1998). These "class-based" surface emissivities are valuable for studies at regional and global scale, but too coarse for local studies. An alternative method is to obtain relative surface emissivity, or "pixel-based" surface emissivity, directly from satellite TIR data. This method does not need ground measurements and is independent of the spatial resolution of the data. It is applicable to surface emissivity estimation from fine spatial resolution satellite data such as Landsat Enhanced Thematic Mapper Plus (ETM+) TIR bands with $60 \times 60 \mathrm{~m}$ pixel size (Yang, 2003).

While there have been comparisons of interpolation methods, little research has been directed towards the assessment and calibration of LST interpolation. Therefore the objectives of this study are to: 1) evaluate the effectiveness of interpolation methods in estimating LST using weather station mea- 


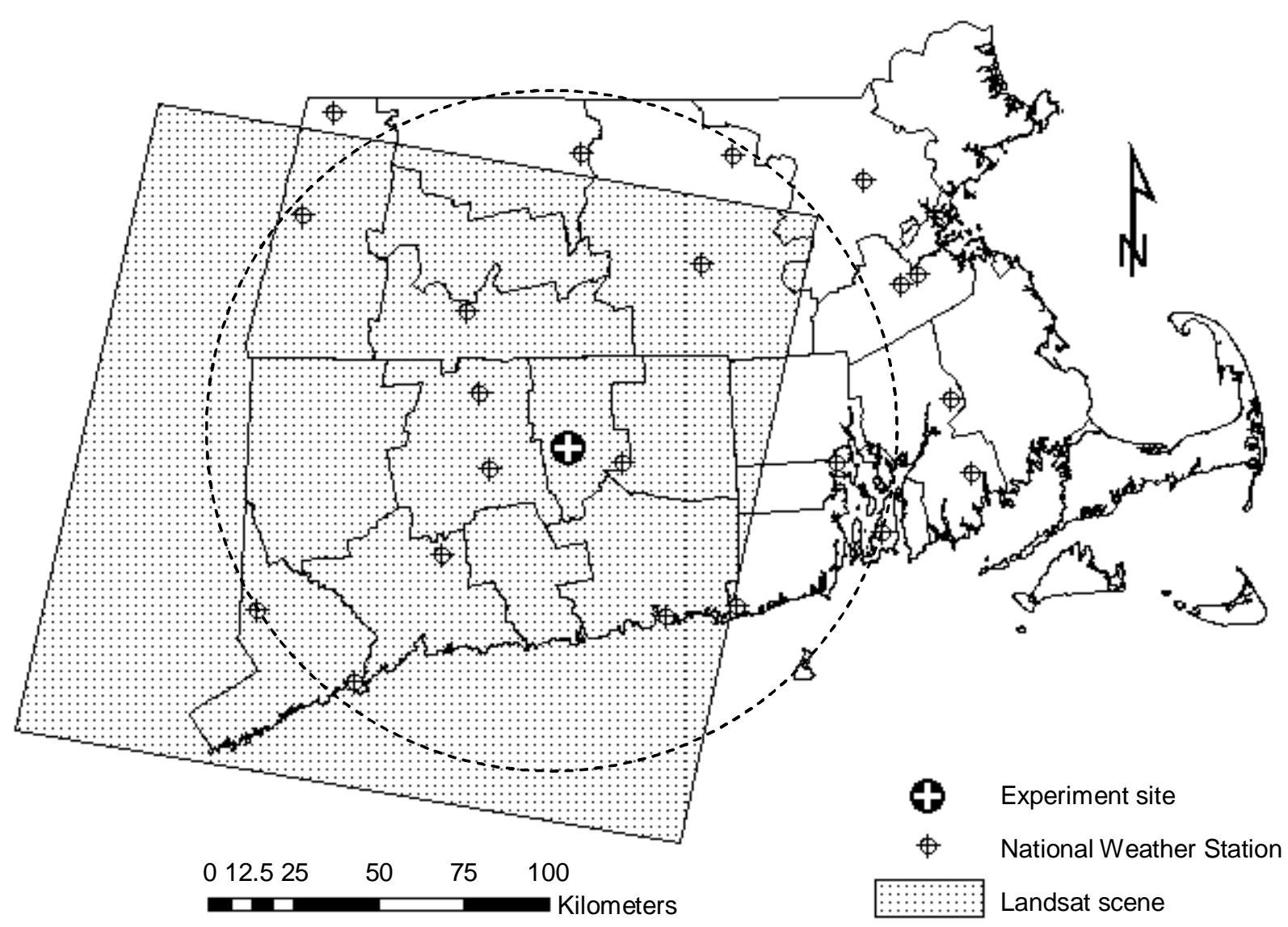

Note: It is a $100 \mathrm{~km}$ buffer around the experimental site from where in situ measurements were collected for validation. The area is mostly covered by one scene (path 13/row 31) of Landsat imagery that was used to derived surface emissivity. Temperature measurements during the study period used for spatial interpolation were retrieved from 13 weather stations in this area.

Figure 1. The study area in southern New England.

surements, and 2) calibrate the LST interpolation using satellite-derived surface emissivity.

\section{Background}

\subsection{Study Area and Data}

The experimental site of this study is an observation tower (Lat. $41^{\circ} 47^{\prime} 30^{\prime \prime} \mathrm{N}$, Long. $72^{\circ} 22^{\prime} 29^{\prime \prime} \mathrm{W}$ ) in a mixed forest in Coventry, Connecticut. This site is flat with 47-year old red maple (Acer rubrum) overstory and occasional white pine (Pinus strobes) and trembling aspen (Populus tremuloides) trees at the edges. The specific boundary of the study area is defined as a $100 \mathrm{~km}$ buffer around the tower (Figure 1). The radius of $100 \mathrm{~km}$ is to ensure that there are at least 10 NWS sites in this area so that spatial interpolations can be conducted.
Field measurements were made at the experimental site between June and September 2001. Air temperatures of the canopy surface were collected using aspirated copper-constantan thermocouples every five seconds and averaged every 30 minutes. Theses data were used to evaluate the effectiveness of each interpolation method and the calibration process using satellite-derived surface emissivity.

There are 13 NWS in the buffer area (Table 1). Hourly surface temperatures at these weather stations during the study period were retrieved from the National Climatic Data Center (NCDC). Temperature measurements of NWS at 10:30 each day during the study period were extracted to generate temperature contour surfaces by different interpolation methods.

Landsat ETM+ image (path 13/row 31) acquired on September 5, 2001 was used to derive surface emissivity for the 
study area. Satellite-derived surface emissivity was then applied to calibrate the temperature interpolation using station measurements in the same day.

Table 1. National Weather Stations from where hourly Temperature Observations were Retrieved from National Climatic Data Center for the Study Period and Used to Interpolate Temperature Surfaces

\begin{tabular}{llllll}
\hline $\begin{array}{l}\text { Station } \\
\#\end{array}$ & City & State & $\begin{array}{l}\text { Elevation } \\
(\mathrm{m})\end{array}$ & $\begin{array}{l}\text { Latitude } \\
\left({ }^{\circ} \mathrm{N}\right)\end{array}$ & $\begin{array}{l}\text { Longitude } \\
\left({ }^{\circ} \mathrm{W}\right)\end{array}$ \\
\hline 1 & Bridgeport & $\mathrm{CT}$ & 1.5 & 41.175 & 73.146 \\
2 & Danbury & $\mathrm{CT}$ & 139.3 & 41.371 & 73.483 \\
3 & Groton & $\mathrm{CT}$ & 3 & 41.328 & 72.049 \\
4 & Hartford & $\mathrm{CT}$ & 48.8 & 41.938 & 72.683 \\
5 & Hartford & $\mathrm{CT}$ & 5.8 & 41.736 & 72.651 \\
6 & Meriden & $\mathrm{CT}$ & 31.4 & 41.510 & 72.828 \\
7 & Willimantic & $\mathrm{CT}$ & 75.3 & 41.742 & 72.184 \\
21 & Orange & MA & 169.2 & 42.570 & 72.291 \\
22 & Pittsfield & MA & 363.9 & 42.427 & 73.289 \\
25 & Westfield & MA & 82.6 & 42.158 & 72.716 \\
26 & Worcester & MA & 300.5 & 42.267 & 71.876 \\
28 & Providence & RI & 15.5 & 41.722 & 71.433 \\
29 & Westerly & RI & 24.7 & 41.350 & 71.799 \\
\hline
\end{tabular}

\subsection{Spatial Interpolation}

The interpolation techniques tested in this study include two deterministic interpolation methods -- IDW and Spline, and two geo-statistical interpolation methods -- Kriging and Cokriging.

IDW is based on the assumption that values close to one another are more alike than those that are farther apart. In other words, those measured values closest to the prediction location will have the most influence on the predicted values than those farther away (Johnston et al., 2001). In IDW, each measured point has a local influence that diminishes with the linear distance between the sampled and unsampled points. How fast the local influence diminishes with the distance is controlled by the parameter called power. If the parameter power equals zero, there is no decrease with distance, the weights will be the same and the prediction will be the mean of all measured values. If the power value is very high, only the immediate few surrounding points have influence on the prediction. With the default power value of two the interpolation method is also called Inverse Square Distance Weighting.

Splining is a commonly used deterministic interpolation method to represent two-dimensional curves on three-dimension surfaces. The Spline method can be considered as fitting a rubber-sheeted surface through the known points using a mathematical function. In ArcGIS, the Spline interpolation is a Radial Basis Function (RBF). The RBFs are used for calculating smooth surfaces from a large number of data points. The Spline function produces good results for gently varying surfaces such as rainfall. Spline is not suitable when there are large changes in the surface values within a short horizontal distance.

In classical statistics, observations at sampled points are assumed independent. That is, there are no correlations between the observations. Kriging is a geostatistical technique similar to IDW in that it uses a linear combination of weights at known points to estimate values at unknown points. Kriging considers the distances between sampled points and their autocorrelation. Kriging uses a semivariogram, a measure of spatial autocorrelation between points, so the weights change according to the spatial arrangement of the samples (Goovaerts, 1997).

Cokriging is a moderately quick interpolator that can be exact or smoothed depending on the measurement error model. Cokriging uses information on more than one variable. Cokriging calculation includes estimating the autocorrelation for each variable as well as all cross-correlations between variables. Cokriging has the following models:

$$
\begin{aligned}
& Z_{1}(s)=\mu_{1}+\varepsilon_{1}(s) \\
& Z_{2}(s)=\mu_{2}+\varepsilon_{2}(s)
\end{aligned}
$$

where $Z_{I}$ is the main variable of interest, representing surface temperature, $Z_{2}$ is the ancillary variable, representing the elevation of weather stations in this study, $\mu_{1}$ and $\mu_{2}$ are unknown constants, $\varepsilon_{1}(s)$ and $\varepsilon_{2}(s)$ are two types of random error. Cokriging uses multiple datasets and allows investigation of both autocorrelations for $Z_{1}$ and $Z_{2}$ and cross-correlation between $Z_{1}$ and $Z_{2}$.

\subsection{Surface Emissivity Derivation and Calibration}

Surface emissivity for a large area is usually derived from classification of satellite imagery and ground measurements (Snyder et al., 1998). Since calibration of the spatial interpolation needs "pixel-based" relative emissivity instead of "class-based" absolute emissivity, we calculate surface emissivity of the study area by its definition, i.e., dividing satellite-derived spectral radiance of each pixel with the spectral radiance of blackbody. Spectral radiance of each pixel was estimated from the digital number $(D N)$ of Landsat ETM+ band 6 (high-gain) pixel values using the following equation (Singh, 1988):

$R=R_{\min }+\frac{\left(R_{\max }-R_{\min }\right) \times\left(D N-D N_{\min }\right)}{255}$

where $R$ is the mean spectral radiance in the spectrum of 10.4 - $12.5 \mu \mathrm{m}\left(\mathrm{W} \cdot \mathrm{cm}^{-2} \cdot \mathrm{sr}^{-1} \cdot \mu \mathrm{m}^{-1}\right), R_{\min }$ and $R_{\max }$ are minimum and maximum scene radiances, and $D N_{\min }$ and $D N_{\max }$ are minimum and maximum pixel digital numbers in the thermal image.

Blackbody is an ideal material that completely absorbs all incident radiation and converts it to internal energy, then emits (re-radiates) the absorbed energy at the maximum possi- 
ble rate per unit area. We calculated blackbody radiance in three ways (Yang, 2003). The first regarded pixels that have maximum digital number in the image as blackbody and used the maximum $D N$ of high-gain thermal band (226) to replace $D N$ in Equation (3). The second assumed a perfect blackbody and used 255 to replace $D N$ in Equation (3). In this case, the blackbody radiance $\left(R_{\max }\right)$ of Landsat $7 \mathrm{ETM}+$ high-gain thermal band has a value of $12.65 \mathrm{~W} \cdot \mathrm{cm}^{-2} \cdot \mathrm{sr}^{-1} \cdot \mu \mathrm{m}^{-1}$ after July 1, 2000 (NASA, 2003). The third replaced the $D N$ in Eq.3 by the average of the maximum $D N$ of the scene and 255. The third one gave the best result and was used in this study.

We calculated surface emissivity for each pixel of the image using map algebra function in Spatial Analyst in ArcGIS software. Derived surface emissivity map was then used to calibrate the spatial interpolation in the following procedure. First, we extract surface emissivities of the pixels where NWS sites are located from satellite-derived surface emissivity. We also retrieved temperature measurements at 10:30 on September 5, 2001 at weather stations from NCDC. Then we transformed measured temperatures to standardized temperatures by dividing them with satellite-derived surface emissivity at each station. We used standardized temperatures at NWS to interpolate LST using Kriging method. Finally we converted interpolated temperature map to surface temperature map by multiplying it with the satellite-derived surface emissivity.

\section{Results and Discussion}

The output of the interpolations includes 348 temperature surfaces for a consecutive 87-day period. Figure 2 illustrates the spatial patterns of temperatures interpolated from NWS measurements on September 5, 2001 using different interpolation methods. Results from IDW showed a typical "cow eyes” pattern in circle extents, indicating its strong local influence of the value at weather stations (Figure 2a). Kriging showed the pattern with consideration of both local influence and spatial autocorrelation of samples (Figure 2c), while Spline showed a smoother pattern (Figure 2b). Cokriging with consideration of elevation resulted in almost the same pattern as that from Kriging (Figure 2d). This is because the ancillary variable in Cokriging, i.e., elevation, has small variations in the study area so that its contribution to the interpolation could be ignored.

We also examined the performance of each interpolation method using scatter plots between predicted temperature and observed temperature at the experimental site (Figure 3). The predicted temperatures are the temperatures at 10:30 each day in summer 2001 extracted from interpolated temperature surfaces. The observed temperatures are in situ measurements on the observation tower. We found that all methods yield high correlations between interpolated and observed temperatures $\left(R^{2}=0.94\right)$ at the experimental site. This indicates that with ground measurements calibration all methods can be used to interpolate LST. Similar results were obtained by examining the root mean square error (RMSE) from cross validation of each interpolation method (Table 2.) Meanwhile we found that, without ground measurements calibration, interpolations alone overestimate an average of $8{ }^{\circ} \mathrm{C}$ at the experimental site. This is probably due to the difference in surface absorption and emittance of radiation between forest surface at experimental site and open space at NWS sampling sites (i.e., airports).

Table 2. Descriptive Statistics of RMSE in Cross Validation of LST Interpolation Using NWS Temperature Measurements at 10:30 for Consecutive 87 Days (July 2 to September 26, 2001)

\begin{tabular}{llllll}
\hline & \multicolumn{5}{c}{ Root-Mean-Square-Error $\left({ }^{\circ} \mathrm{C}\right)$} \\
& IDW & Spline & Kriging & Cokriging & $\mathrm{N}$ \\
\hline Mean & 2.091 & 1.851 & 2.056 & 1.923 & 87 \\
Median & 2.029 & 1.78 & 1.986 & 1.828 & 87 \\
Minimum & 1.048 & 0.482 & 0.924 & 0.72 & 87 \\
Maximum & 4.738 & 4.892 & 4.947 & 4.524 & 87 \\
Standard Deviation & 0.611 & 0.685 & 0.642 & 0.692 & 87 \\
\hline
\end{tabular}

Figure 4 shows the daily variation of interpolation error of IDW on September 5, 2001. The interpolation error ranges from $4.1{ }^{\circ} \mathrm{C}$ to $19{ }^{\circ} \mathrm{C}$ with an average of $10{ }^{\circ} \mathrm{C}$. Highest interpolation error occurred at hour 07:00 in the morning. The error decreased after 07:00 and reached the lowest at around 18:00. The reason could be that solar radiation received by different land surfaces tends to be saturated in the late afternoon. The significant variation of interpolation error indicates that the time of data collection is also an important factor in LST estimation. Daily minimum interpolation error of $4{ }^{\circ} \mathrm{C}$ also indicates that spatial interpolation alone is not sufficient to estimate accurate LST without further calibrations.

Figure 5 is the surface emissivity of the study area derived from Landsat high-gain TIR image. We used high-gain TIR data for the reason that it has a large range of brightness values and gives more accurate temperature estimation in most situations. Through calibration using satellite-derived surface emissivity, the average difference between interpolated and observed temperature reduced from $10{ }^{\circ} \mathrm{C}$ to $1.56{ }^{\circ} \mathrm{C}$. The improvement in interpolation by surface emissivity is because that most NWS sites are located in open areas such as airports, and the interpolation method is based on the assumption that the study area has a homogeneous land surface with similar surface emissivity.

\section{Conclusions}

For a specific location that has ground measurements available for calibration, any of the four interpolation methods discussed could be used to interpolate surface temperature. For a region with sparsely field measurements, the calibration method using satellite-derived surface emissivity presented in this study is a good option in estimation of accurate LST. For 


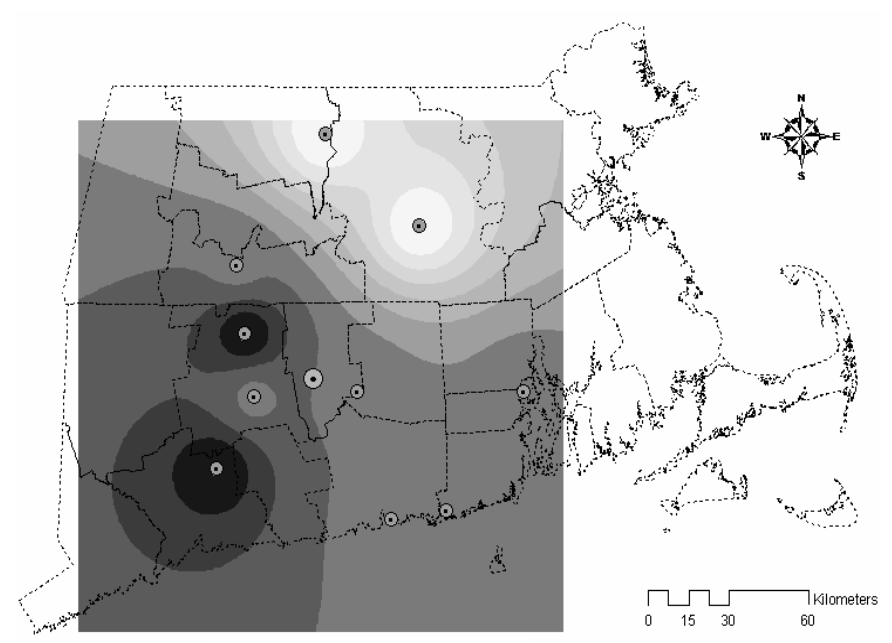

a. IDW

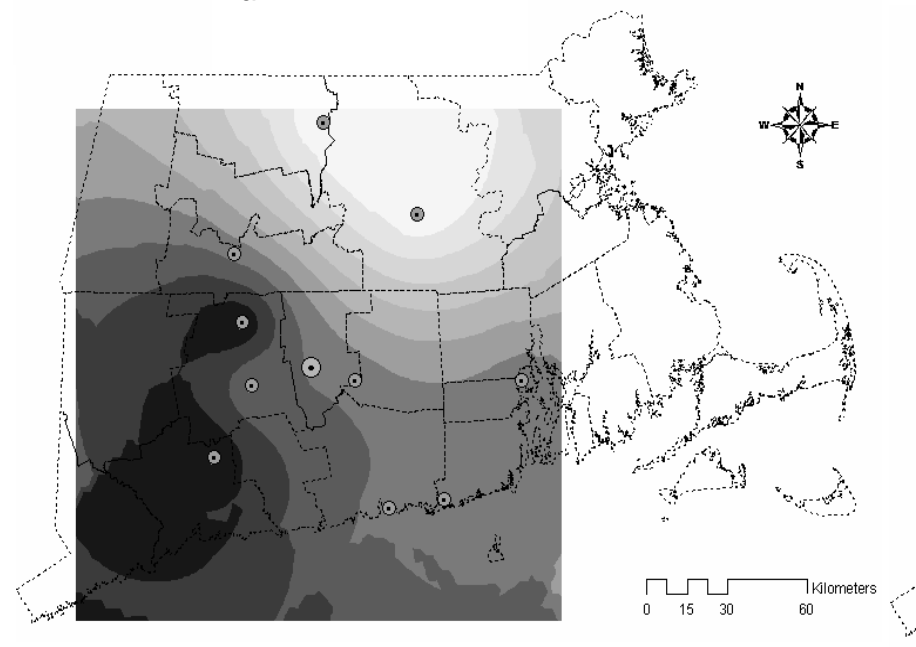

c. Kriging

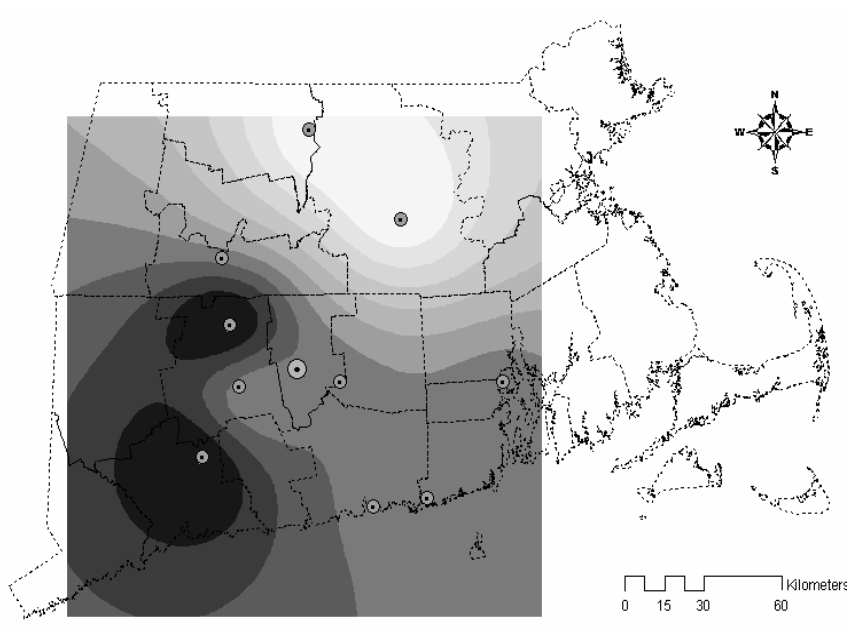

b. Spline

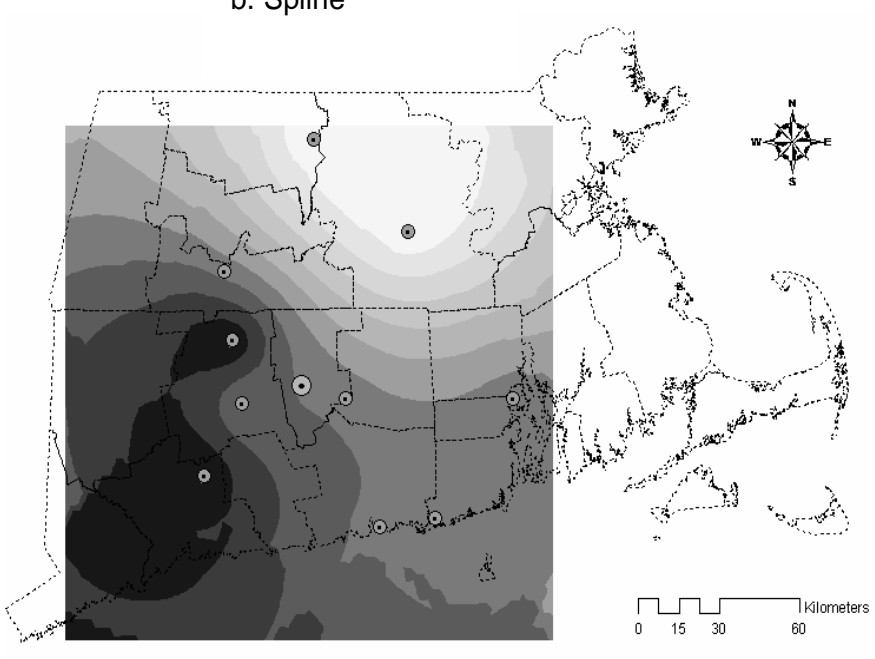

d. Cokriging

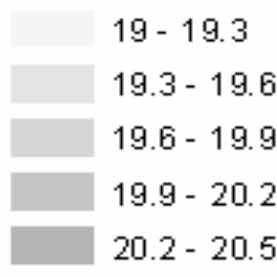

$20.5-20.8$

20.8 - 21.1

$21.1-21.4$

$21.4-21.7$

$21.7-22$

Figure 2. Temperature surfaces patterns $\left({ }^{\circ} \mathrm{C}\right)$ interpolated by IDW, Spline, Kriging, and Cokriging using temperature measurement at 10:30 on September 5, 2001 retrieved from national weather stations. 
IDW Interpolation

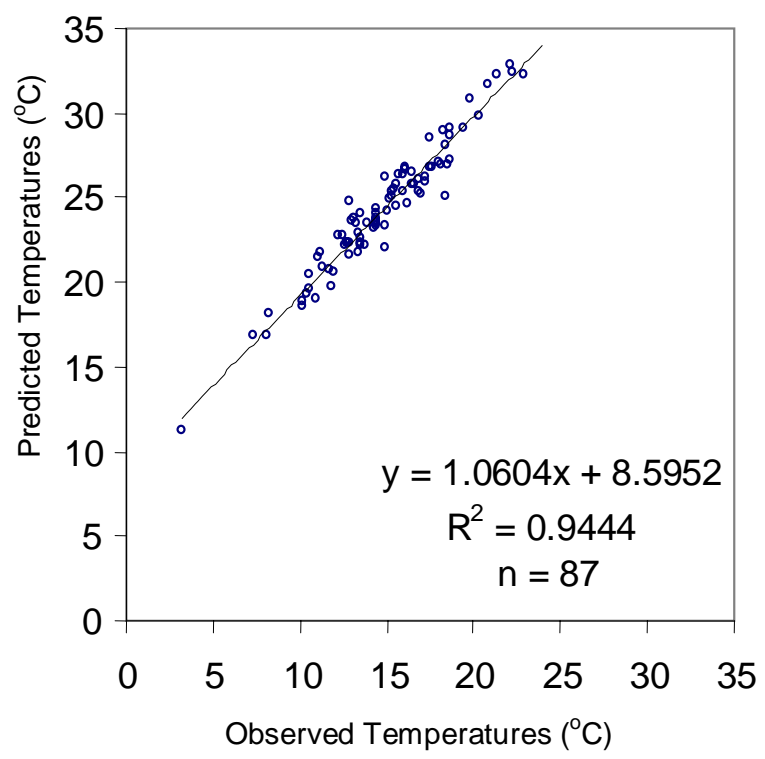

Ordinary Kriging Interpolation

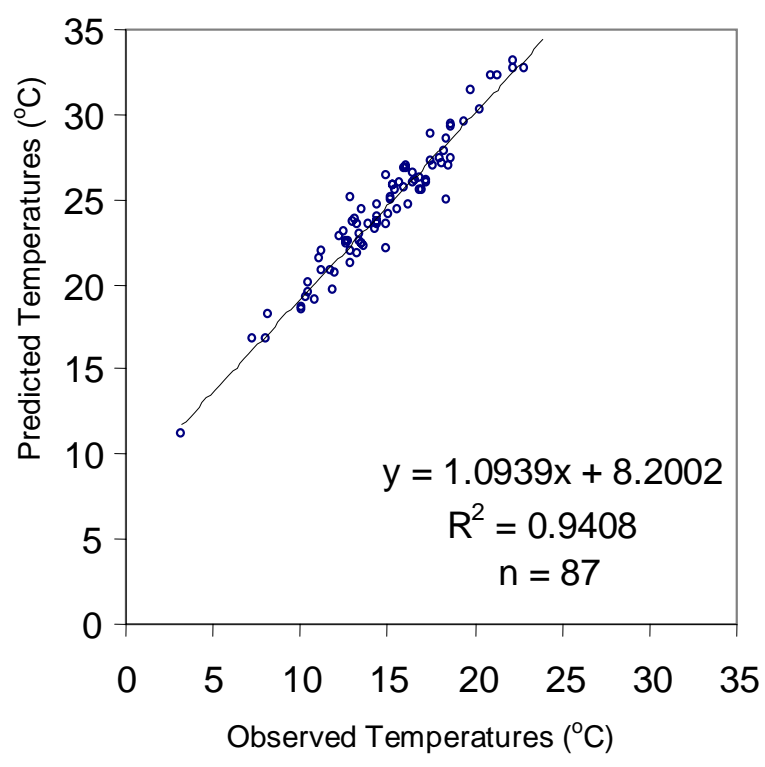

Spline Interpolation

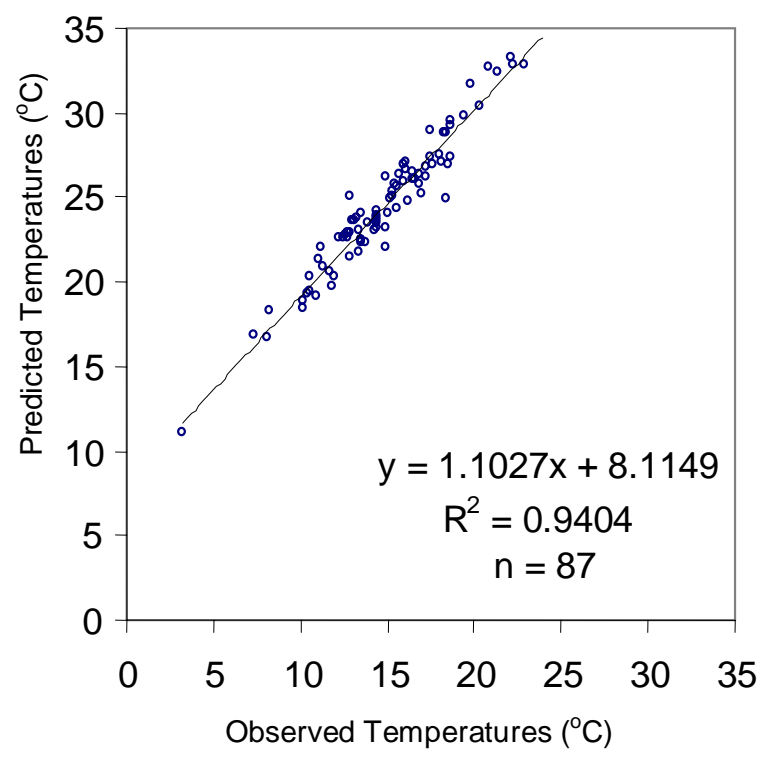

Ordinary Cokriging Interpolation

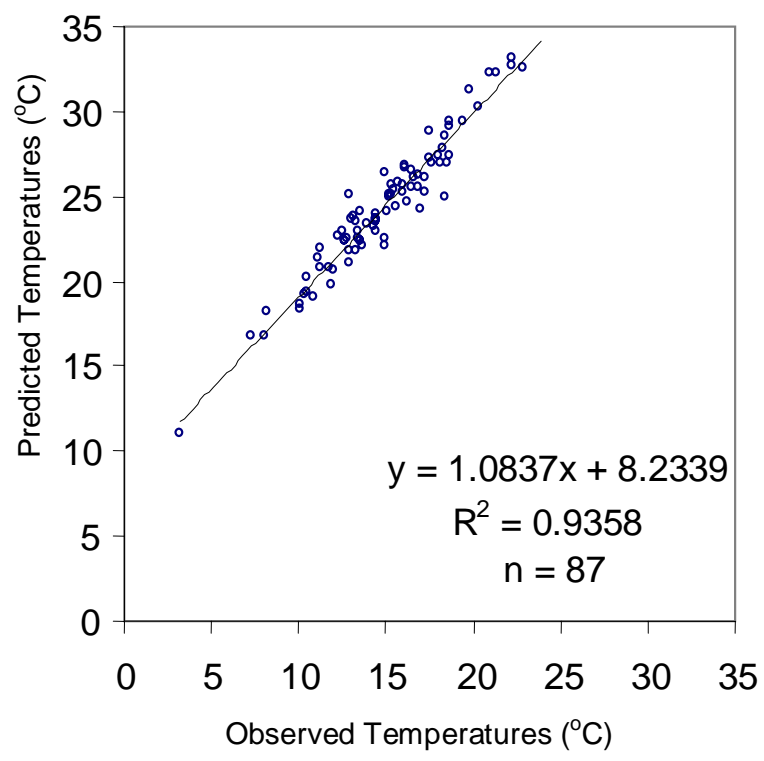

Figure 3. Scatter plots between observed temperatures $\left({ }^{\circ} \mathrm{C}\right)$ every day at 10:30 during July 2 and September 26 in 2001 at the experimental site and predicted temperature $\left({ }^{\circ} \mathrm{C}\right)$ using four different inteprolation methods. 


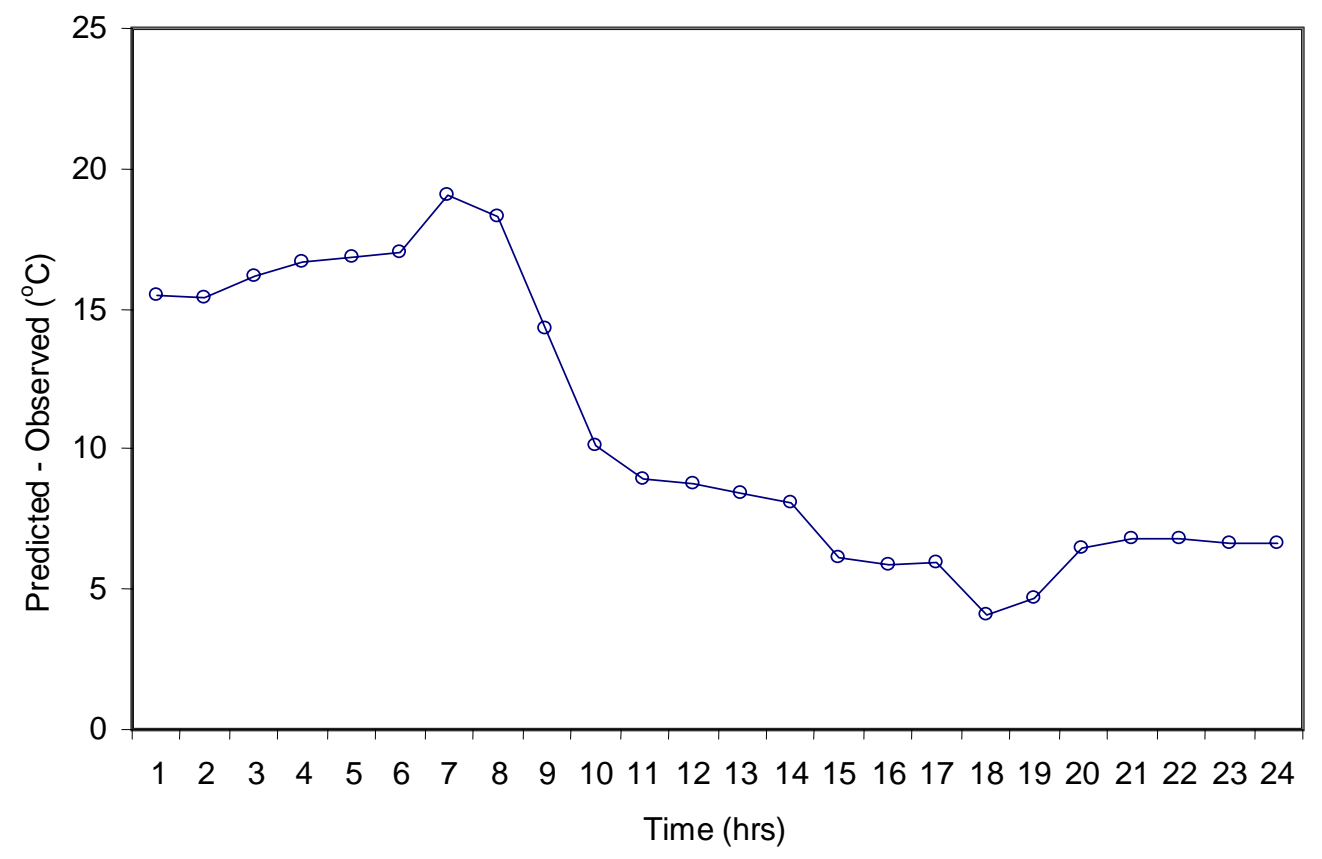

Figure 4. Daily variation of the prediction error $\left({ }^{\circ} \mathrm{C}\right)$ of IDW at the experimental site on September 5, 2001 using original temperature measurements from weather stations.

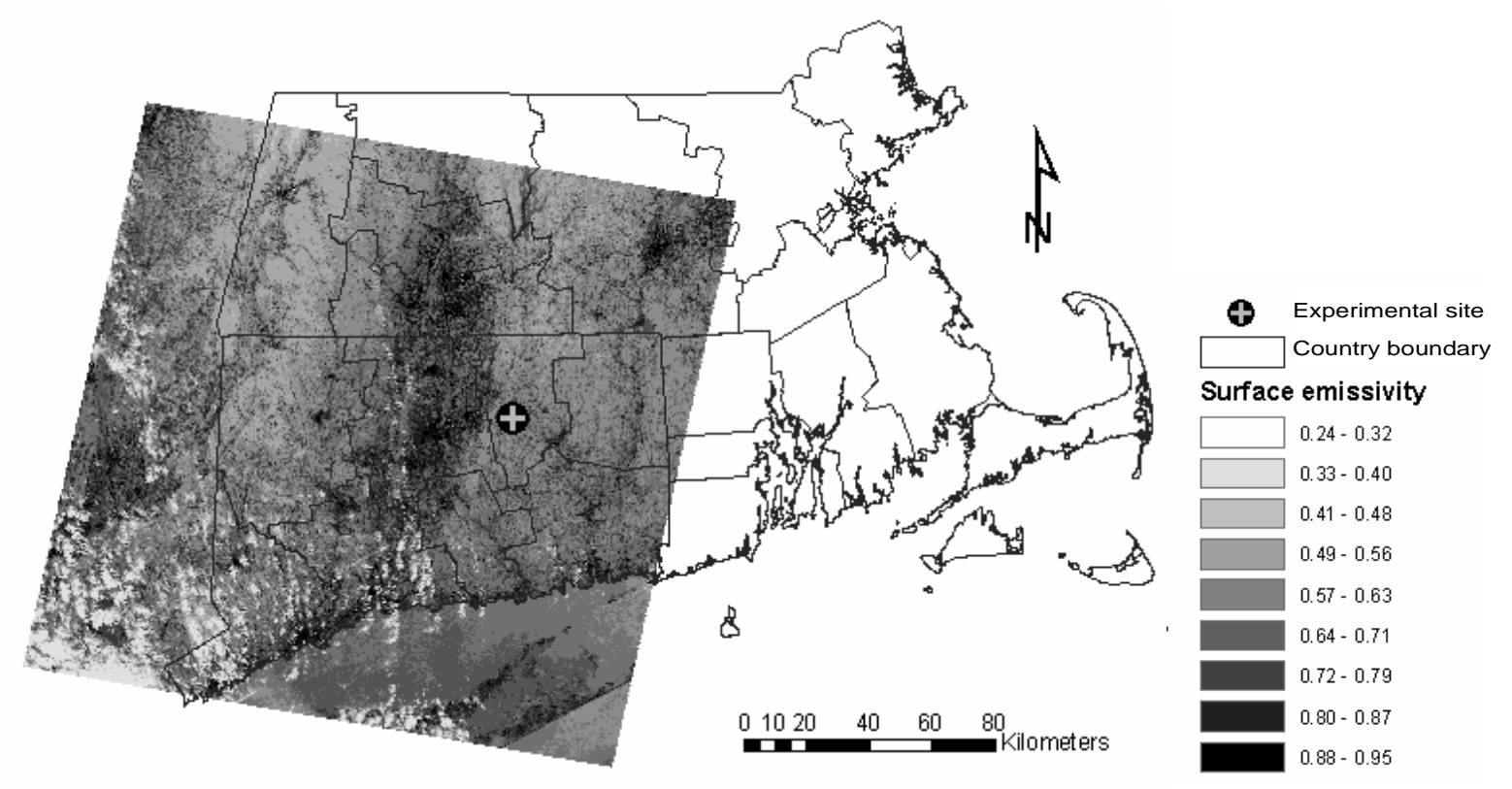

Figure 5. Surface emissivity map derived from Landsat ETM+ TIR data acquired on September 2, 2001. 
a region without surface emissivity or satellite TIR data, Kriging interpolation is recommended due to its considerations of prediction confidence in error map and spatial autocorrelation between sampling sites. Cokriging is suggested for areas with rough terrains and large variation in elevations. We recommended that our method of combining spatial interpolation and satellite-derived surface emissivity for LST estimation be tested in more diverse landscapes for validations.

Acknowledgments. This research was funded by NASA (Grant No.NAG5-8739). The authors appreciate the support by Dr. David Miller of University of Connecticut for allowing us to use the observation tower and field measurement equipment.

\section{References}

Goovaerts, P. (1997). Geostatistics for Natural Resources Evaluation. New York, Oxford University Press, pp. 483.

Hughes, D.A. (1982). The relationship between mean annual rainfall and physiographic variables applied to a coastal region of southern Africa. South Afr. Geogr. J., 64, 41-50.

Ishida, T. and Kawashima, S. (1992). Use of cokriging to estimate surface air temperature from elevation. Theor. Appl. Climatol., 47, 147-157.

Johnston, K., Ver Hoef, J.M., Krivoruchko, K. and Lucus, N. (2001). Using ArcGIS Geostatistical Analyst. Redlands, ESRI, pp. 300.

Lefohn, A.S., Knudsen, H.P. and McEvoy, Jr.L.R. (1988). The use of kriging to estimate monthly ozone exposure parameters for the southeastern United States. Environ. Pollut., 53, 27-42.

Lindkvist, L. and Lindqvist, S. (1997). Spatial and temporal variabil- ity of nocturnal summer frost in elevated complex terrain. Agr. Forest Meteorol., 87, 139-153.

NASA. Landsat 7 Science Data Users Handbook (United States). http://lpwww.gsfc.nasa.gov/IAS/handbook/handbook_toc.html (accessed Oct. 1, 2003).

Phillips, D.L., Dolph, J. and Marks, D. (1992). A comparison of geostatistical procedures for spatial analysis of precipitation in mountainous terrain. Agr. Forest Meteorol., 58, 119-141.

Price, D.T., McKenney, D.W., Nalder, I.A., Hutchinson, M.F. and Kesteven, J.L. (2000). A comparison of two statistical methods for spatial interpolation of Canadian monthly mean climate data. Agr. Forest Meteorol., 101 (2-3), 81-94.

Qin, Z. and Karnieli, A. (1999). Progress in the remote sensing of land surface temperature and ground emissivity using NOAA-AVHRR data. Int. J. Remote Sens., 20(12), 2367-2393.

Singh, S.M. (1988). Brightness temperature algorithms for Landsat Thematic Mapper data. Remote Sens. Environ., 24, 509-512.

Snyder, W., Wan, Z., Zhang, Y. and Feng, Y.Z. (1998). BRDF models to predict spectral reflectance and emissivity in the thermal infrared. IEEE Trans. Geosci. Remote Sens., 36 (1), 214-225.

Vizuete, W., Junquera, V., MaDonald-Buller, E., McGaughey, G., Yarwood, G. and Allen, D. (2002). Effects of temperature and land use on predictions of biogenic emissions in Eastern Texas, USA. Atmos. Environ., 36, 3321-3337.

Wahba, G. (1981). Spline interpolation and smoothing on the sphere. SIAM J. Sci. Stat. Comput., 2(1), 5-15.

Willmott, C., Robeson, S. and Philpot, W. (1985). Small-scale climate maps: A sensitivity analysis of some common assumptions associated with grid-point interpolation and contouring. Am. Cartographer, 12(1), 5-16.

Yang, J. (2003). Remote Sensing Modeling of Land Surface Temperature, Ph.D. Dissertation, College of the Environment and Life Sciences, University of Rhode Island, Kingston, RI, USA. 